\title{
25 Research Square \\ Effect of Cardiopulmonary Bypass Machine on the Platelet Function Testing.
}

Khalid A. AlSaleh

King Saud University Medical City

Rashed B. AlBakr

King Saud University Medical City

Turki B. AlBacker

King Saud University College of Medicine

Rakan AINazer

King Saud University College of Medicine

Abdulkareem Almomen

King Saud University Medical City

Osama T. Khoja

King Saud University College of Medicine

Tarek Owaidah ( $\sim$ bmujahid@gmail.com )

King Faisal Specialist Hospital and Research Center

\section{Research article}

Keywords: Cardio-Pulmonary Bypass Machine, PFA-100, platelet dysfunction, Multiplate Electrode Aggregometry

Posted Date: August 3rd, 2020

DOI: https://doi.org/10.21203/rs.3.rs-45350/v1

License: (c) (i) This work is licensed under a Creative Commons Attribution 4.0 International License.

Read Full License 


\section{Abstract}

Background: Bleeding during coronary artery bypass surgery is a leading cause of mortality. Several factors have been associated with bleeding, platelet dysfunction being the most significant.

Objective: to assess the effect of cardiopulmonary bypass machine (CPB) during cardiac surgery on platelet function using Platelet Function Analyzers (PFA-100), and Multiplate Electrode Aggregometry (MEA), and correlating that with a drop in Hemoglobin $(\mathrm{Hb})$.

Methods: Whole blood samples were collected preoperative and sixty minutes intraoperatively of different cardiac procedures utilizing (CPB) and tested for platelet function by PFA-100 and MEA. Complete blood count was measured using an automated hematology analyzer.

Results: A significant difference was found between pre- and intraoperative ADP and EPI measurement in PFA-100, where preoperative PFA-ADP values displayed the ability to predict the intra-op drop in $\mathrm{Hb}(P-$ value 0.01 , correlation coefficient 0.4699 ). At the same time, pre-op MEA-Ristocetin and TRAP showed an inverse correlation with an intra-op drop in $\mathrm{Hb}(-0.31$ and -0.36$)$.

Conclusion: The current study reported significant changes in platelet dysfunction in cardiac surgeries with $\mathrm{CPB}$, measured by two modalities PFA-100, and MEA. While PFA-100 and MEA both detected the changes in platelet dysfunction due to CPB, PFA-100 results were sensitive and positively predicted intraop $\mathrm{Hb}$ drop as compared to MEA. There was a significant change in $\mathrm{Hb}$ one hour into the CPB, indicating that platelet transfusion might help decrease Intra- and postoperative bleeding independent of the platelet count as they are dysfunctional. PFA-100 results can be relied upon for distinction of high-risk cardiac surgery patients for bleeding and can be used for clinical decision making to improve patient outcome.

\section{Introduction:}

Conflicting reports exist in the literature concerning the role of Platelet function analyzer (PFA-100) in identifying high-risk patients for bleeding during $\mathrm{CPB}(1)$; ranging from high negative to high positive predictive values in cardiac surgery patients for postoperative transfusion management (2). Multiple factors play a role related to bleeding during $\mathrm{CPB}$, which could be due to the operative procedure or the operator and type of procedure. Platelet dysfunction as an entity by itself could be one of the causes of haemorrhage. The estimation of the exact diagnostic performance of PFA-100 could determine its utility for clinical decisions. Multiplate Electrode aggregometry (MEA) is another modality used for rapid on-site detection of platelet dysfunction. A worldwide survey of 37 countries and 202 laboratories reported that PFA and MEA are used for screening of platelet function disorders in $53 \%$ and $12 \%$ of laboratories, respectively(3). This extensive use, in turn, leads to clinical decisions which determine patient outcome. PFA-100 is used more excessively than MEA for the screening of primary hemostatic disorders; however, a meta-analysis showed that the majority of studies reporting diagnostic accuracy and sensitivity of PFA100 lacked quality and reporting requirements, impeding precise characterization of its clinical utility (1). 
Thus, emphasizing the need for evidence-based critical evaluation of the diagnostic performance of PFA100 and MEA for platelet dysfunction in the clinical setting.

Several other factors are linked to morbidity and mortality associated with CPB, but none singularly has been precisely proven(4). The routine procedure of CPB still requires immediate therapeutic improvements and optimization strategies to deal with pre-and intraoperative complications, such as functional haemostatic defects leading to its hall-mark of bleeding(5). Bleeding incidence after CPB varies from $5-25 \%$, and platelet dysfunction is possibly the most plausible yet controversial explanation for coagulopathy related to $\mathrm{CPB}(6)$. Platelets, the smallest anucleate fragments in the blood, are activated during CPB and play a crucial role in hemostasis and immune response, specifically systemic inflammation, tissue repair, and regeneration $(7,8)$. Quantitative, as well as qualitative aspects of platelet dysfunction, participate in thromboembolism and microvascular injury(9). Platelets communicate and build a number of interactions with a variety of blood proteins like coagulation factors and endothelium, thus imparting paramount contribution in hemostasis, directly and indirectly(10). However, in CPB related hemostatic defects, functional abnormalities of platelets are extrinsic rather than intrinsic. This is depicted by optimal platelet reactivity in CPB in-vitro and a minimum amount of degranulated circulating platelets(11).

Owing to the paramount importance of platelet function in bleeding diathesis with its subsequent influence on therapeutic strategies and patient outcome, there is a dire need to address two major aspects of cardiac surgery. Firstly, the different modalities of cardiac surgery utilizing CPB and its effect on platelet function; and secondly the evaluation of the diagnostic performance of point-of-care testing devices dedicated to the measurement of platelet function, such as PFA-100, MEA, and VerifyNow System $(2,12-15)$.

The current study aimed to evaluate two methods PFA-100 \& MEA side by side comparing and identifying which one is more sensitive in predicting the risk of intra-op bleeds. We presumed that the use of sensitive, time saving and efficient test for prediction of high-risk patients would govern accurate clinical decisions, leading to better patient outcome and reduced mortality associated with CPB.

\section{Methods:}

Patient selection: The study was approved by the Institutional Review Board of College of Medicine, King Saud University, Kingdom of Saudi Arabia. Samples were collected after informed consent indicating the purpose of the study and the right of the participant to withdraw at any time without any obligation towards the study team. Blood samples from $(n=34)$ adult patients were recruited from KKUH, having routine normal coagulation tests prior to the different cardiac procedures, including valve replacement, coronary artery bypass etc. were collected at two points in time; at the beginning of the CPB and 60 min into CPB. All patients going for CPB procedure were advised to stop antiplatelet therapy five days prior to the procedure. 
Before starting the CPB for every patient, $20 \mathrm{cc}$ blood from central-line access point was collected for complete blood count (CBC), and platelet function tests. The second time-point was 60 min after the start of CPB. The two blood samples at each point in time were collected as one sample in the EDTA tube for $\mathrm{CBC}$, and in the Lithium tube for platelet function tests. Citrated blood in sodium citrate tubes was aspirated. Platelet and blood products were transfused as needed as per the discretion of the cardiac surgeon.

Platelet function testing: PFA-100 analyzer and MEA were used to measure the extent of platelet dysfunction pre- and intra- $\mathrm{CPB}$. Complete blood counts including HCT, Hb and PLT counts were measured using a hematology analyzer (Advia 120; Bayer, Tarrytown, NY, USA), and platelet function analysis by PFA-100 (Siemens Healthcare Diagnostics, Malvern, PA, USA). Disposable cartridges on high shear rates were used for PFA-100, containing aperture inside a membrane coated with cADP or cEPI, which induced platelet activation leading to adhesion and aggregation, resulting in occlusion of aperture. The time needed to occlude aperture and stop the blood flow is called Closure Time (CT). The longer is the closure time; the more platelet dysfunction is expected(16).

Whole blood samples from these patients were also tested by a Multiplate analyzer (Dynabyte, Munich, Germany). Aggregation and adhesion of platelets on electrode surfaces are converted to electrical impedance which is measured by Multiplate analyzer. The test cuvette contained platelet agonists; aspirin (ASP: $15 \mathrm{mM}),($ TRAP: $1 \mathrm{mM})$, collagen (COL) $(100 \mathrm{lg} / \mathrm{mL})$, arachidonic acid (ADP $0.2 \mathrm{mM})$, and ristocetin (RISTO: $10 \mathrm{mg} / \mathrm{mL}$ ). Platelet adhesion after activation results in aggregation on metal sensor causing electrical impedance between electrode wires, which was measured continuously for six minutes and analyzed after conversion to arbitrary units.

We calculated the most important parameter, which is the area under the aggregation curve (AUC). It is affected by the total height of the aggregation curve as well as by its slope and is best suited to express the overall platelet activity, and expressed as AUC*min(14). Lower value indicates more platelet dysfunction

\section{Definitions}

Thrombin receptor-activating peptide (TRAP-6); a potent platelet activator and stimulates platelet aggregation via stimulation of platelet thrombin receptors. Aspirin or Clopidogrel cannot block this action. This means TRAP allows Gpllb/llla antagonists to be detected even in samples containing Aspirin (ASP) or Clopidogrel. Adenosine diphosphate (ADP) stimulates platelet activation by the ADP receptors. In COL test, Collagen activates the collagen receptor, which in turn activates the platelets via arachidonic acid production. Ristocetin (RISTO) platelet aggregation test is based on the binding of RISTO-vWF (von Willebrand factor) complexes leading to the aggregation of platelets as well as their activation. Aspirin ${ }^{\circledR}$ inhibits this activation. This explains the ASP sensitivity of RISTO test.

\section{Data Analysis}


Descriptive statistics were computed as a baseline means, standard deviations (SD) and minimum and maximum values for continuous variables. Preoperative variables were compared to intra-operative variables for PFA-100 and MEA. We used skewness-kurtosis normality tests to find variables with nonnormal distribution. Student t-test compared significance in mean values of normally distributed variables, while the Wilcoxon t-test compared significance in mean values of non-normally distributed variables. We used the software STATA v.13.0 (Stata Corp., College Station, TX, USA) in our analysis. A statistical significance threshold of $P<0.05$ was adopted.

\section{Results}

Our study compared the effect of CPB on platelet function by using pre-and intra-operative platelets function parameters for 34 patients using PFA-100 analyzer and MEA. Data for two patients were removed due to erroneous PFA-100 values leading to 32 patients' data used for this study. Although all patients going for CPB procedure were advised to stop antiplatelet therapy five days prior to the procedure, we found 7 patients presenting with high PFA-100, suggesting some residual effect of antiplatelet.

Out of 32 patients, $9(28.1 \%)$ were females with a mean age of $53.1 \pm 15.4$ (m: 52 , r: $30-73$ years), and 23 (71.9\%) were male with a mean age of $58.9 \pm 8.75$ ( $\mathrm{m}: 59, \mathrm{r}: 40-79$ years), where ' $\mathrm{m}$ ' denotes the median, and ' $r$ ' denotes range.

We presumed that pre- and intra-operative measurements should show significant change towards worsening of platelet function. Pre-and intra-operative PFA-100, MEA and CBC measurements are summarized in Table-1. The variables showing greatest pre and intra-op variability are depicted as a Box Whisker plot in Figure-1, where one set depicts both PFA- ADP and epinephrine (EPI), and other shows only MEA-ADP and RISTO. The ADP observations for pre-and intra-op are 32 and 31 respectively, with an increase in mean values from $125.9 \pm 69.9$ (m: 102, r: $54-300 \mathrm{sec}$ ) to $205 \pm 74.9$ (m: 162 , r:79-300). The EPI measurements also showed an increase in mean values from $166.8 \pm 72.5(\mathrm{~m}: 187, \mathrm{r}: 64-300)$ to $214.2 \pm 42.7$ ( $\mathrm{m}: 210, \mathrm{r}: 115-300)$. This increase clearly shows worsening of platelet function. The significance of platelet function change is estimated using student t-test, both ADP and EPI show significant difference $P$-values $<0.001$ and 0.002 , respectively.

Table-1 also summarizes MEA measurements. Overall, the tests show a decline in the measurements consistent with a decline in the aggregation(17). A significant decline $(P$-value $<0.001)$ in mean and median values of ADP (AUC) is observed from $81.5 \pm 28.3$ ( $\mathrm{m}: 75.5$, r: 26-124) to $56.8 \pm 27.4$ (m: 53.5 , r: 7-39 AUC*min; a 30\% decrease). A decline in ASP was insignificant ( $P$-value 0.085$)$ in mean and median values from $59.2 \pm 27.6$ (m: 56.5 , r: $23-122)$ to $50.2 \pm 30.8$ (m: 48.5, r: 10-112 AUC*min; a $14 \%$ decrease). A decrease in COL was significant ( $P$-value 0.002$)$ in mean and median values from $50 \pm 19$ (m: 43.5, r: 27-97) to $37.1 \pm 18.1$ (m: 35; r: 4-80 AUC*min; a $24.5 \%$ decrease). Similarly, a significant decline was observed in RISTO and TRAP mean and median values ( $P$-value 0.0005 and 0.02 , respectively). RISTO decreased from $76.8 \pm 30.9$ (m: 72.5, r: 33-156 AUC*min) to $50.9 \pm 37.9$ (m: 41, r:4- 
181 AUC*min; a 33.7\% decrease). TRAP declined from $114 \pm 26.3$ (m: 112, r: 51-178) to $100 \pm 36$ (m: 95 , r: 13-179 AUC*min, a 3.5\% decrease). This little change in TRAP indicates that the sample is not contaminated with antiplatelets (14).

A significant drop in $\mathrm{Hb}$ values was also observed one hour after the start of $\mathrm{CPB}(P-$ value $<0.001)$ from $137 \pm 12.5$ (m: 11.6, r: 71-157) to $8.43 \pm 1.24$ (m: 8.3, r: 9-108 g/L, a 26\% decrease); HCT ( $P-$ value < 0.001 ) from $34.5 \pm 5.5$ (m: 34.2, r: 23.4-46.7) to $25 \pm 2.6$ (m: 24.5, r:20.6-29.7; a 27.5\% decrease); PLT $(P-$ value $<0.001)$ from $264 \pm 131.9(\mathrm{~m}: 255)$ to $185.3 \pm 112.6(\mathrm{~m}: 164)$.

Next, we tried to answer if PFA-100 pre-op values related to intra-op drop in $\mathrm{Hb}$ value, as in, do we expect lower $\mathrm{Hb}$ count for elevated PFA-100 values. We assumed two approaches to find the answer: first, we look for a correlation between PFA-100 variables and intra-op Hb; secondly, we run a statistical t-test which shows a significant change in $\mathrm{Hb}$ groups based on a dichotomy of PFA-100 variables. The binary variable was labelled 'abnormal' when both ADP and EPI values exceeded the normal range and labelled 'normal' otherwise.

We used Pearson's correlation and found a weak correlation coefficient between PFA-ADP and intra-op drop in $\mathrm{Hb}$ (0.4699), which is illustrated as a scatter plot in Figure-2. On the other hand, pre-op EPI showed no correlation (0.21) with the drop in intra-op Hb values, as illustrated in Figure-3. For t-test, we created a binary variable based on the values of PFA-ADP and EPI. Next, we partitioned the post-op drop in $\mathrm{Hb}$ based on this binary variable and compared their means using t-test. The number of patients in the abnormal set was 6 , and 26 in the normal set. The difference of mean turned out to be significant $(P-$ value 0.01), and $\mathrm{Hb}$ mean values for abnormal set $75 \pm 0.5$ (m: 75, r:69-82) were lower than that of the normal set $86 \pm 1.02$ (m:87, r:71-108). An observation worth mentioning is that for MEA test, only pre-op RISTO and TRAP showed a weak inverse correlation with the intra-op drop in $\mathrm{Hb}(-0.31$ and -0.36$)$, while rest of the MEA variables didn't.

Another perspective was to look at the change in intra-op PFA-100 and MEA values against a drop in intra-op $\mathrm{Hb}$. Change in intra-op PFA-100 and MEA values were obtained by subtracting intra-op from preop values. Similarly, the drop in intra-op $\mathrm{Hb}$ value was obtained by subtracting intra-op $\mathrm{Hb}$ from pre-op $\mathrm{Hb}$. For PFA-100, change in intra-op ADP was weakly correlated to the drop in intra-op Hb (0.46). For MEA, intra-op change in ASP was weakly correlated to the drop in intra-op $\mathrm{Hb}(0.41)$.

Considering the gender, mean $\mathrm{Hb}$ levels in female patients $75 \pm 0.5(\mathrm{~m}: 73, \mathrm{r}: 69-84 \mathrm{~g} / \mathrm{L})$ were significantly lower (a 16\% decrease, $P$-value 0.0009 ) than the male patients $87 \pm 1.0$ (m: $87, \mathrm{r}: 71-$ $108 \mathrm{~g} / \mathrm{L})$. The same pattern was observed in HCT levels.

\section{Discussion}

Bleeding during cardiac surgery procedures makes allogeneic blood transfusion an essential requirement to maintain hemostatic blood components(13); therefore, accurate and timely assessment of patients in need of transfusion is crucial. To meet these requirements, evaluation of platelet function along with 
platelet count intra-operatively is pivotal. PFA-100 and MEA are two commonly used modalities to monitor platelet function and assess the need for transfusion and blood flow cessation(18). Intraoperative transfusions play a crucial role in the patient outcome; therefore, sensitive and efficient platelet function test should be performed for predicting high-risk patients. The current study compared the predictive value and sensitivity of PF-100 and MEA for the identification of high-risk intra-operative patients using the intra-operative drop in $\mathrm{Hb}$ as a reference outcome.

The PFA-100® works on the principle of shear stress during vascular injury and provides quantitative data related to high shear force-associated platelet dysfunction with whole blood, and provides accurate and quantitative in-vitro measurements $(18,19)$. In the current study, a significant difference $(P<0.05)$ in platelet function was observed pre- and intra-CPB through PFA-ADP (125.9 \pm 69.9 vs $205 \pm 74.9)$, as well as EPI measurements $(166.8 \pm 72.5$ vs $214.2 \pm 42.7)$ with an increase in platelet dysfunction after starting $\mathrm{CPB}$. However, conflicting results have been reported previously regarding intra-operative bleeding and its association with platelet dysfunction assessed by PFA-100(20). In agreement with our findings, a positive relationship between the extent of bleeding and platelet dysfunction was reported by Ostrowsky et al.(21) and Raman et al.(22). On the other hand, and contrary to our findings, Fattorutto et al. (23) and Forestier et al.(24) proved that PFA-100 results couldn't distinguish patients at low risk of bleeding from patients who had significant bleeding; excluding the high-risk CPB patients. In cardiothoracic procedures involving CPB, specificity and sensitivity of PFA-100 were $94 \%$ and $85 \%$, respectively, in identifying response to platelet transfusion. Our PFA-100 results clearly predicted the outcome based on preoperative analyses as measured by correlation with intra-op drop in $\mathrm{Hb}$ values via Pearson correlation. Beside the Pearson co-relation test, we used a different yet meaningful approach to distinguish high and low-risk patients by generating binary variables based on values of PFA-100 ADP and EPI. The binary variable is considered abnormal when both ADP and EPI values were out of normal range. This approach is useful as some of the platelet inhibitors such as aspirin and Clopidogrel affect EPI during the test. In such a scenario, there was a significant difference between $\mathrm{Hb}$ levels of abnormal and normal groups.

MEA is another frequently used modality for platelet function analysis, which can be opted for the nearpatient setting to get prompt results. MEA has certain advantages over PFA-100, including measurement of platelet function in whole blood in the presence of other blood cells for timely assessment(25). Although, in conformity with PFA-100 results, significant changes in platelet function were observed through MEA in pre- and intra-CPB, as indicated by a decreased aggregation depicted through less area under the curve. Meyer et al. showed the significance of MEA performed at the start of a surgical procedure to improve the management of blood product transfusion(26). A positive correlation between the preoperative values of ADP from MEA and total blood transfusion during surgical intervention was reported in the study of Mengistu et al.(27). However, in the current study, we found the diagnostic performance of MEA as comparatively weaker than that of PFA-100, taking an intra-op drop in $\mathrm{Hb}$ as an outcome. The role of MEA in lowering the number of transfusions has not been justified previously in the literature. Apropos, in the current study, MEA couldn't provide such benefits due to lower sensitivity as compared to PFA-100. Contrary to the previous studies(3), we found that PFA-100 is sensitive to detect the changes in platelet dysfunction and can be relied upon to distinguish high-risk patients for bleeding. 
PFA-100 also showed high variability pre-and intra-CPB in ADP and EPI; while in MEA, only ADP and RISTO show greater variability intra CPB as compared to pre-CPB values; depicting the higher sensitivity level of PFA-100.

The novelty of the current study lies in the fact that intra-operative transfusion management has been studied considering the intra-op drop in $\mathrm{Hb}$ as a primary outcome, which is easy to measure on-site as compared to calculations based on units transfused post cardiac surgery. Limitations of the current study lie in the fact that MEA and PFA-100 results have not been compared with the gold standard platelet function test: Light transmission aggregation(3).

This study does have a limitation of correlating the bleeding to drop in the $\mathrm{Hb}$ after 60 minutes of the procedure, which might reflect fluid priming the CPB or retrograde priming. Still, the timing of this measurement might be of value to limit the variability and complexity of different procedures, affect surgeons' preferences and allow the analysis of platelet dysfunction in a broader range of cardiac procedures.

\section{Conclusion}

The current study reported significant worsening in the platelet function 60 min intra-operatively during CPB, measured by two modalities PFA-100 and MEA, which might be reversed by platelet transfusion during surgery. We found PFA-100 to be more sensitive and predictive of intra-op drop in $\mathrm{Hb}$ as compared to MEA,

\section{Abbreviations}

Adenosine diphosphate (ADP); Aspirin (ASA); Epinephrine (EPI). Cardiopulmonary Bypass (CPB); Coronary Artery Bypass Graft (CABG); Platelet Function Analyzer (PFA); Multiplate Electrode Aggregometry (MEA)

\section{Declarations}

Authors' Contribution: KS \& TO designed \& developed the study. Both authors were responsible for contents \& authenticity. RB, TB, RN, OK oversaw data collection, data entry, final review of data and analysis, were responsible for the direction of the study team, and facilitation of the project plan.

Funding and Source of Support: Deanship of Scientific Research, King Saud University, Researcher Supporting Project Number RSP-2019/88 for Dr. Khalid AISaleh.

Ethics approval and consent to participate: The study was approved by the Institutional Review Board of College of Medicine, King Saud University, Kingdom of Saudi Arabia.

Consent for publication: All authors consent for publication. 
Availability of data and materials: Furnished upon request.

Competing interests: None declared.

Acknowledgement: Nazia Sadaf, MD, for her contribution in medical writing.

\section{References}

1. Karger R, Donner-Banzhoff N, Müller H-H, Kretschmer V, Hunink M. Diagnostic performance of the platelet function analyzer (PFA-100®) for the detection of disorders of primary haemostasis in patients with a bleeding history-a systematic review and meta-analysis. Platelets. 2007 Jun;18(4):249-60.

2. Paniccia R, Priora R, Liotta AA, Abbate R. Platelet function tests: a comparative review. Vasc Health Risk Manag. 2015 Feb;18:11:133-48.

3. Moenen FC, Vries MJ, Nelemans PJ, van Rooy KJ, Vranken JR, Verhezen PW, et al. Screening for platelet function disorders with Multiplate and platelet function analyzer. Platelets. 2019;30(1):81-7.

4. Welsby IJ, Bennett-Guerrero E, Atwell D, White WD, Newman MF, Smith PK, et al. The association of complication type with mortality and prolonged stay after cardiac surgery with cardiopulmonary bypass. Anesth Analg. 2002 May;94(5):1072-8.

5. Höfer J, Fries D, Solomon C, Velik-Salchner C, Ausserer J. A snapshot of coagulopathy after cardiopulmonary bypass. Clin Appl Thromb Hemost. 2016 Sep;22(6):505-11.

6. Varghese S, Unni M, Mukundan N, Rai R. Platelet functions in cardiopulmonary bypass surgery. Med J Armed Forces India. 2005 Oct;61(4):316-21.

7. Xu XR, Zhang D, Oswald BE, Carrim N, Wang X, Hou Y, et al. Platelets are versatile cells: New discoveries in hemostasis, thrombosis, immune responses, tumor metastasis and beyond. Crit Rev Clin Lab Sci. 2016 Dec;53(6):409-30.

8. Katz JN, Kolappa KP, Becker RC. Beyond thrombosis: the versatile platelet in critical illness. Chest. 2011 Mar;139(3):658-68.

9. Weerasinghe A, Taylor KM. The platelet in cardiopulmonary bypass. Ann Thorac Surg. 1998 Dec;66(6):2145-52.

10. Elzey BD, Tian J, Jensen RJ, Swanson AK, Lees JR, Lentz SR, et al. Platelet-mediated modulation of adaptive immunity: a communication link between innate and adaptive immune compartments. Immunity. 2003 Jul;19(1):9-19.

11. Kestin AS, Valeri C, Khuri S, Loscalzo J, Ellis P, MacGregor $H$, et al. The platelet function defect of cardiopulmonary bypass. Blood. 1993 Jul;82(1)(1):107-17.

12. Albanyan A, Al-Musa A, AlNounou R, Al Zahrani H, Nasr R, AlJefri A, et al. Diagnosis of Glanzmann thrombasthenia by whole blood impedance analyzer (MEA) vs. light transmission aggregometry. Int J Lab Hematol. 2015 Aug;37(4):503-8. 
13. Angiolillo D, Levy J, Schneider D, Jennings L. The Role of Platelet Function Testing in Improving Clinical Outcomes. The Medical Roundtable Cardiovascular Edition. 2020 Jan 18.

14. Calatzis A, Spannagl M, Loreth R. Multiplate ${ }^{\circledR}$ platelet function analysis-Application and interpretation; 2007. Available from URL (cited 22.06. 2015): http://www. ecomeds. ru/data/files/catalog/1.\% 20Compendium\% 20Multiplate\% 20final\% 20V2. 0_07. 2007.

15. Gibbs N. Point-of-care assessment of antiplatelet agents in the perioperative period: a review. Anaesth Intensive Care. 2009 May;37(3):354-69.

16. Bogdanic D, Karanovic N, Mratinovic-Mikulandra J, Paukovic-Sekulic B, Brnic D, Marinovic I, et al. The Role of Platelet Function Analyzer Testing in Cardiac Surgery Transfusion Management. Transfus Med Hemother. 2017 Apr;44(2):106-13.

17. Hanke A, Roberg K, Monaca E, Sellmann T, Weber C, Rahe-Meyer N, et al. Impact of platelet count on results obtained from multiple electrode platelet aggregometry (Multiplate). Eur J Med Res. 2010 May 18;15(5):214-9.

18. Koltai K, Kesmarky G, Feher G, Tibold A, Toth K. Platelet aggregometry testing: Molecular mechanisms, techniques and clinical implications. Int J Mol Sci. 2017 Aug 18;18(8):1803.

19. Valsami S, Kollia M, Mougiou V, Sokou R, Isaakidou E, Boutsikou M, et al. Evaluation of PFA-100 closure times in cord blood samples of healthy term and preterm neonates. Clin Chem Lab Med. 2020 Mar;26(4):e113-6. 58(.

20. Favaloro EJ. Clinical utility of closure times using the platelet function analyzer-100/200. Am J Hematol. 2017 Apr;92(4):398-404.

21. Ostrowsky J, Foes J, Warchol M, Tsarovsky G, Blay J. Plateletworks ${ }^{\mathrm{TM}}$ Platelet Function Test Compared to the Thromboelastograph ${ }^{\mathrm{TM}}$ for Prediction of Postoperative Outcomes. J Extra Corpor Technol. 2004 Jun;36(2):149-52.

22. Raman S, Silverman NA. Clinical utility of the platelet function analyzer (PFA-100) in cardiothoracic procedures involving extracorporeal circulation. J Thorac Cardiovasc Surg. 2001 Jul;122(1):190-1.

23. Fattorutto M, Pradier O, Schmartz D, Ickx B, Barvais L. Does the platelet function analyzer (PFA-100) predict blood loss after cardiopulmonary bypass? Br J Anaesth. 2003 May;90(5):692-3.

24. Forestier F, Coiffic A, Mouton C, Ekouevi D, Chene G, Janvier G. Platelet function point-of-care tests in post-bypass cardiac surgery: are they relevant? Br J Anaesth. 2002 Nov;89(5):715-21.

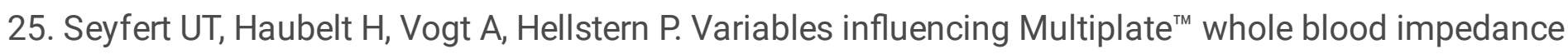
platelet aggregometry and turbidimetric platelet aggregation in healthy individuals. Platelets. 2007 May;18(3):199-206.

26. Rahe-Meyer N, Winterhalter M, Boden A, Froemke C, Piepenbrock S, Calatzis A, et al. Platelet concentrates transfusion in cardiac surgery and platelet function assessment by multiple electrode aggregometry. Acta Anaesthesiol Scand. 2009 Feb;53(2):168-75.

27. Ranucci M, Baryshnikova E. The interaction between preoperative platelet count and function and its relationship with postoperative bleeding in cardiac surgery. Platelets. 2017 Dec;28(8):794-8. 
Figures

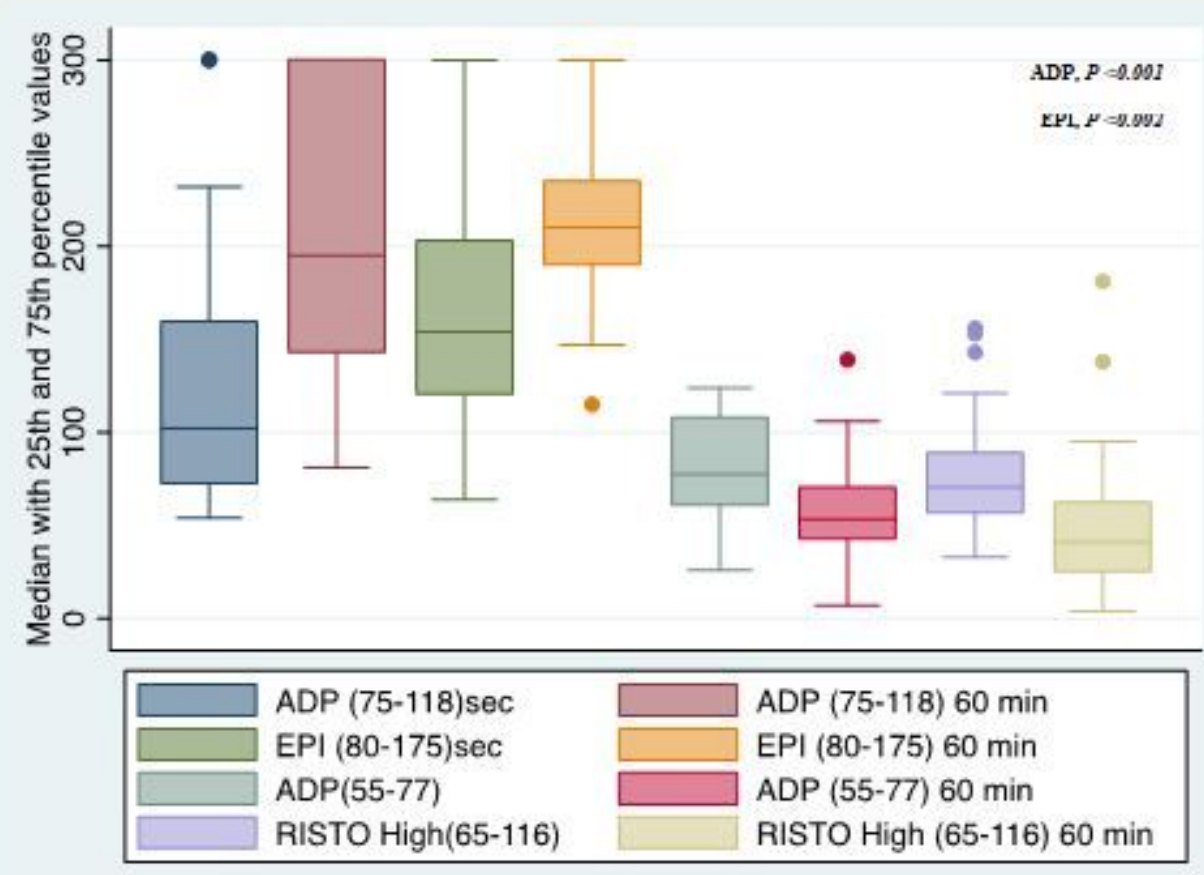

\section{Figure 1}

Box Whisker plot: on the left shows high variability of both PFA-ADP and EPI, and on the right shows only MEA-ADP and RISTO with high variability.

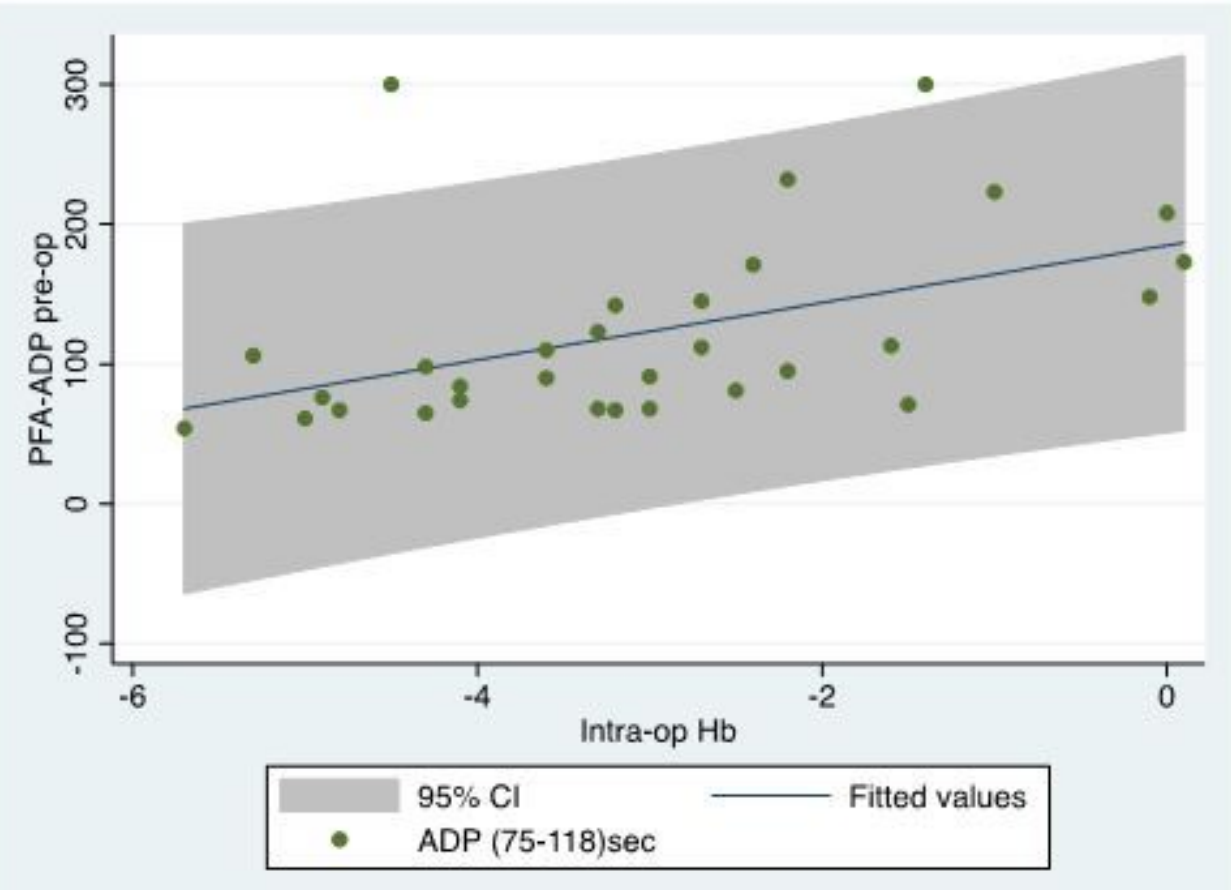

Figure 2 
Scatter plot between pre-op PFA-100 ADP and intra-op drop in Hb values.

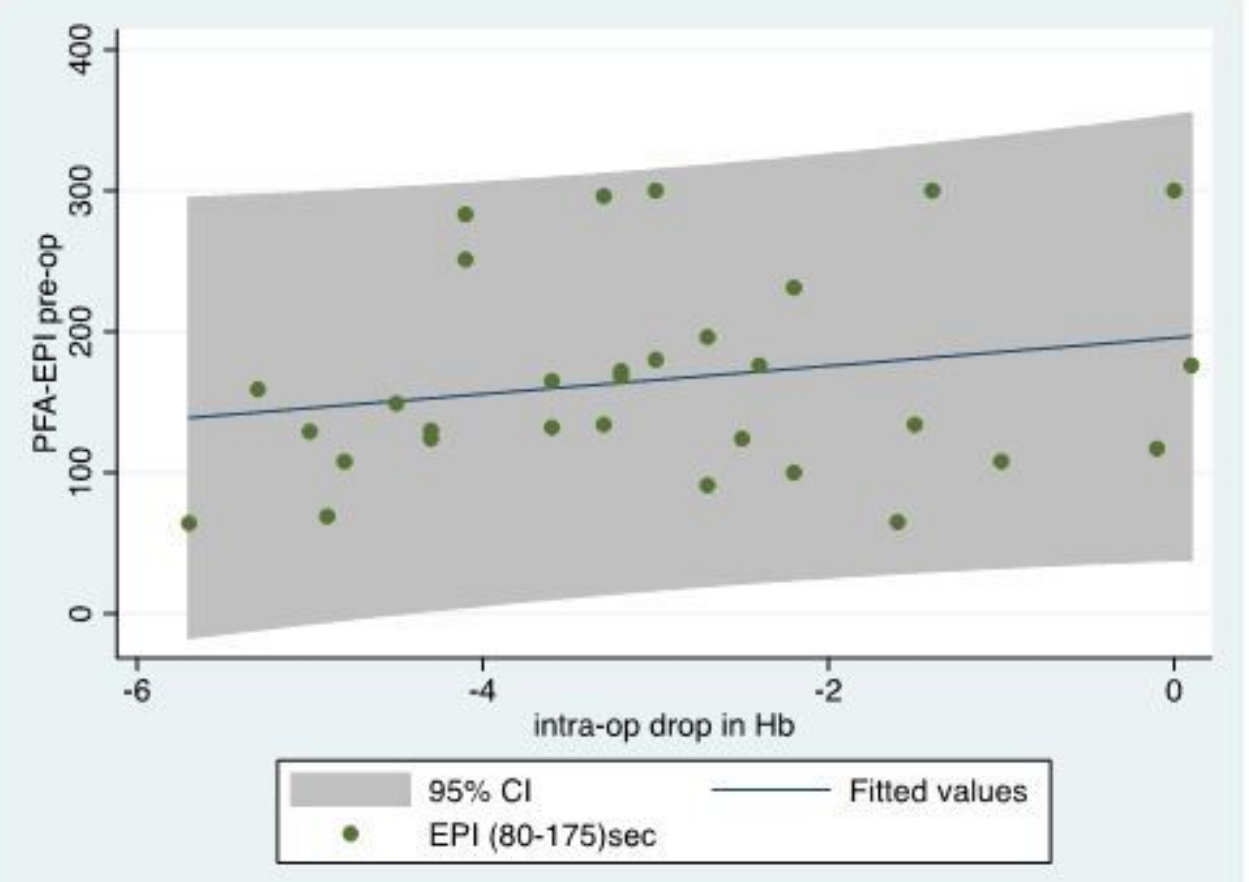

Figure 3

Scatter plot between pre-op PFA-100 EPI and intra-op drop in Hb values. 